\title{
TRASCENDENCIA DEL TRIGO RECOLECTADO POR LA EXPEDICIÓN DE MOPOX EN CUBA (1797-1799)
}

\author{
Rolando E. Misas Jiménez
}

\section{RESUMEN}

La expedición de Mopox recolectó en su estancia en la isla de Cuba, una variedad de trigo aclimatado que constituía una rareza de la flora agrícola de La Habana. La labor recolectora realizada por el naturalista expedicionario Baltasar Boldo, favoreció la descripción que Mariano Lagasca realizó más tarde en el Real Jardín Botánico de Madrid. El esfuerzo de los botánicos españoles ha servido para identificar el trigo que continuó cultivándose en la región de Villa Clara en el siglo XIX, y para recomendar a las instituciones agrícolas cubanas el cultivo de un tipo de trigo cercano al que encontró Boldo en las cercanías de La Habana.

\section{SUMMARY}

The expedition of Mopox gathers, during their stay in the island of Cuba, a variety of acclimatized wheat that constituted a rarity of the agricultural flora of The Habana. The gathering work carried out by the naturalist and expedicionary Baltasar Boldo, favored the description that Mariano Lagasca fulfill later in the Royal Botanical Garden from Madrid. The effort of the Spanish botanists has served in order to identify the wheat that continued 
cultivating in the region of Villa Clara in the XIXth Century, and in order to recommend to the agricultural Cuban institutions the cultivation of a type of wheat similar to that which Boldo found in the vicinities of The Habana.

\section{Una evidencia de antiguo trigo cubano en el Real Jardín Botánico de Madrid}

Una de las mayores dificultades para estudiar los antiguos cultivos de trigo en Cuba venía dada por la obtención de datos que permitieran descifrar, con el mayor rigor científico posible, la especie de trigo que se diseminó con éxito por determinadas regiones de esa isla antillana. El desconocimiento que existía hacia la exploración botánica de Baltasar Manuel Boldo, como primer botánico de la expedición del conde de Mopox, no contribuía a esclarecer esa incógnita.

Esa dificultad fue superada gracias a la información suministrada por el libro Los trigos de la Ceres Hispánica de Lagasca y Clemente, publicado en Madrid en $1952^{1}$. Sus autores, los ingenieros agrónomos Ricardo Téllez y Manuel Alonso, tuvieron el mérito de engrandecer, luego de haber revisado los herbarios y descripciones de trigos del Real Jardín Botánico de Madrid, el esfuerzo mancomunado que, en distintos momentos de sus vidas, realizaron los botánicos españoles Baltasar Manuel Boldo y Mariano Lagasca en función de un ejemplar de trigo cubano que se cultivó en esos años de dominio español en la isla.

Como resultado de la labor de Boldo y Lagasca, Téllez y Alonso pudieron ofrecer, por primera vez, la única valoración científica de un antiguo Triticum cubano que haya sido realizado por dos reconocidas autoridades de la sistemática botánica de la época.

El ejemplar de trigo recolectado por Boldo en La Habana entre 1797 y 1799 dio por sí mismo una importancia extraordinaria a sus herborizaciones en Cuba ya que facilitó la posterior motivación de Lagasca hacia la descripción y clasificación de ese raro ejemplar de trigo.

A pesar de que el trigo cubano fue un objeto de estudio de personalidades científicas como Boldo y Lagasca, el hecho de que Lagasca definiera ese ejemplar como una nueva especie justifica las dudas que expre-

\footnotetext{
1 Téllez Molina, R.; Alonso Peña, M. (1952), Los trigos de la Ceres Hispánica de Lagasca y Clemente, Madrid, p. 43-44.
} 
saran Téllez y Alonso con respecto al origen y a la cantidad de trigo recolectado por Boldo en Cuba:

Boldo, efectivamente, herborizó en la isla de Cuba, pero es raro que sólo fuese este el único ejemplar hallado y, más raro aún, no habiéndose citado América como origen de ninguna especie de trigo ${ }^{2}$.

\section{Las circunstancias en que Boldo realizó la colecta de trigo en La Habana y no en Villa Clara}

En los momentos en que el zaragozano Baltasar Manuel Boldo, primer botánico de la expedición del conde de Mopox, herborizaba en los alrededores de la capital cubana entre julio de $1797^{3}$ y el día de su fallecimiento, el 31 de julio de 1799, el cultivo del trigo había desaparecido prácticamente en esa zona. La competencia de la harina extranjera y del pan confeccionado con yuca amarga habían afectado el desarrollo del cultivo de ese cereal en La Habana. Por consiguiente, Boldo debió aceptar el ejemplar de trigo que le obsequiara un tal Balth porque lo consideró una rareza de la flora habanera ${ }^{4}$.

Sin embargo, en su recorrido desde Santiago de Cuba hasta La Habana, Boldo debió observar que en la región de Villa Clara el trigo se cultivaba como caso excepcional en la isla. A pesar de que ese trigo contribuía a la subsistencia de las familias campesinas de esa región, Boldo no recolectó ningún ejemplar de esa gramínea villaclareña.

Durante ese recorrido, el conde de Mopox dirigió los trabajos científicos de la expedición de acuerdo con los intereses de los hacendados habaneros reunidos en la Real Sociedad Patriótica de La Habana y en el Real Consulado de Agricultura y Comercio desde que surgieron estas instituciones en 1793 y 1795. En ese sentido, el afán de riqueza que

\footnotetext{
2 Misas Jiménez, R. E. (1991), «La Real Sociedad Patriótica de La Habana en el rescate de la variedad "naturalizada" del trigo de Villa Clara». Asclepio. Monográfico Ciencia y Sociedad en Cuba, 43, 2, p. 124.

3 De acuerdo con Pedro Pablo Herrera existe la certidumbre de que en esa fecha la expedición se encontraba en La Habana (véase: HerRera Oliver, P. P. (1993) «Sobre la Protoflora Cubana, Cubensis Prima Flora y el Herbario de Boldo y Estévez», Fontqueria, Madrid, vol. XXXVI, p. 154.

${ }^{4}$ Misas JimÉnEZ, R. E. (1991) p. 125.
} 
había generado el auge azucarero en La Habana motivaba en Mopox la atención preferente por los recursos agrícolas de otras regiones que tuvieran las posibilidades de aportar la materia prima necesaria para una producción manufacturera en la capital que fuera igualmente libre de ser exportada.

De acuerdo con esos planes agrocomerciales se excluían aquellos cultivos que debían satisfacer las necesidades alimenticias de la población, con lo cual se restringía la curiosidad botánica de Boldo por el trigo de Villa Clara. En gran medida, ese desinterés de Mopox por el trigo se debía a que había obtenido de Manuel Godoy, Príncipe de la Paz, el monopolio de las harinas provenientes de los Estados Unidos de América. A ese lucrativo negocio se hallaba vinculado Francisco de Arango y Parreño, «la más preclara figura antimonopolista de la colonia», añadiéndose a la lista el mencionado Godoy y Carlos Martínez de Irujo, embajador de España en Norteamérica ${ }^{5}$.

El antimonopolismo de los hacendados habaneros contra los comerciantes españoles, por ser éstos los representantes de la nueva política imperial de la monarquía española, fue olvidado cuando tuvieron la oportunidad de arrebatarles el negocio harinero con la complicidad de algunos altos funcionarios del gobierno español. El espíritu de lucro pudo más que la previsión por alcanzar un desarrollo pleno e independiente que permitiera a la economía cubana sustituir las importaciones.

De esta forma, la harina norteamericana se convirtió en el más importante renglón alimenticio de La Habana debido al notable aumento de la población esclava, al abandono parcial de los cultivos de mantenimiento y la gran masa de circulante provocada por el azúcar, que aseguraba la venta de esa harina a cualquier precio. Dado este éxito, Mopox estaba más interesado porque la harina monopolizada llegara a todas las regiones de la isla; por lo tanto, no podía apoyar el desarrollo triguero de Villa Clara ni tampoco podía permitir que Boldo divulgara la existencia de una variedad de trigo aclimatada.

Luego de la fiscalización efectuada por Mopox a las colectas de material botánico en Villa Clara, Boldo debió disfrutar de una mayor libertad de acción en La Habana. El conde de Mopox tenía entonces motivos suficientes para no atender frecuentemente los trabajos de la expedición:

\footnotetext{
${ }^{5}$ Moreno Fraginals, M. (1978), El ingenio, t. 1, p. 100.
} 


\section{TRASCENDENCIA DEL TRIGO RECOLECTADO POR LA EXPEDICIÓN DE MOPOX EN CUBA}

\section{Primer motivo:}

Se había producido un cambio notable en la actitud de los hacendados hacia el propósito agrobotánico del proyecto de expedición de Mopox. Dejaron de pensar en que Boldo y el joven habanero José Estévez contribuyeran a la instalación de una cátedra de botánica y de un vivero de plantas agrícolas que fungieran como Escuela Práctica de Agricultura para los campesinos ${ }^{6}$. En su lugar, le concedieron más importancia al estudio de la naturaleza química de los organismos vegetales ya que preveían la incesante importación de tecnologías manufactureras dedicadas a la extracción de sustancias orgánicas procedentes de productos agrícolas cubanos. En ese sentido, la creación de la Escuela de Química y Botánica, promovida por Nicolás Calvo de la Puerta desde 1793, alcanzó la mayor preferencia ${ }^{7}$. El propio Mopox había asumido, en coordinación con los ensayos de instalación de una máquina de vapor inglesa que iniciara el 11 de enero de 1797 en su ingenio Seibabo, la tarea de traducir la obra de tecnología azucarera de Dutrone de la Couture aprovechando su dominio del idioma francés y sus "notorios conocimientos en química» ${ }^{8}$.

\section{Segundo motivo:}

El inicio de la guerra entre España e Inglaterra el 18 de agosto de 1796 llevó a que Mopox y sus socios temieran un bloqueo comercial marítimo que afectara el disfrute del monopolio harinero. Introdujeron rápidamente, entre el 28 de julio de 1797, fecha en que se sabe con certeza que Boldo se encontraba en La Habana, y el 16 de agosto de 1798, los 47.389 barriles de harina que abarrotaron el mercado habanero produciendo una grave crisis con la harina en mal estado. El Intendente Pablo José Valiente tuvo que emplear el ejército para reprimir las protestas. En definitiva, hubo que tirar al mar 3. 680 barriles de harina inser-

${ }^{6}$ Desde que el hacendado Juan Manuel O'Farrill presentara en 1793 su proyecto de Escuela de Agricultura (véase: ANónimo (1793) «Asuntos que se han tratado en las Juntas ordinarias de la Sociedad», Memorias de la Sociedad Patriótica de La Havana, t. 1, p. 33), se había hecho necesario realizar una enseñanza práctica de la botánica agrícola en los sembrados del futuro vivero, combinándola con descripciones morfológicas sencillas y el conocimiento de los nombres vulgares de los ejemplares sembrados o recolectados.

7 Bachiller y Morales, A. (1965), Apuntes para la historia de las letras y de la instrucción pública en la isla de Cuba, La Habana, t. 1, p. 131.

8 Moreno Fraginals, M. (1978), t. 1, p. 76, 87. 


\section{ROLANDO E. MISAS JIMÉNEZ}

vible pero el Intendente Valiente salvó la situación de Mopox cargándoselos a las Reales Cajas?.

Tercer motivo:

- Boldo no estaba profesionalmente habituado a realizar la actividad botánica con el sentido de utilidad práctica que demandaban los intereses habaneros. Tampoco parecía estar capacitado para formar a José Estévez en los conocimientos fitoquímicos. Todo lo contrario, la solidez de sus conocimientos teóricos respondían al desarrollo que había alcanzado la botánica taxonómica y sistemática durante el siglo XVIII. En esa dirección instruyó a Estévez, para garantizar los resultados provenientes del inventario de los vegetales colectados para su remisión a los herbarios del Real Jardín Botánico matritense, ya que las propias inconsecuencias de los hacendados contribuyeron a que no se priorizara el surgimiento de un vivero de plantas en la isla que estuviera en función de su desarrollo agrícola.

Estas realidades dieron lugar a que Mopox no se ocupara totalmente de la expedición desde su llegada a La Habana. Más bien se dedicó a glorificar la labor teórica o sistemática que habían estado desempeñando Boldo y Estévez para hacerla equiparable o superior a los éxitos obtenidos por la metrópoli con el envío de expediciones a sus dominios ultramarinos como la que había efectuado Martín de Sessé a Nueva España.

El cambio de mentalidad de Mopox al apoyar los trabajos científicos de índole teórico, y no sólo los de utilidad práctica, debió favorecer las iniciativas de Boldo con respecto a la recogida de material botánico. En ese contexto, Boldo adquirió el raro ejemplar de trigo habanero aunque lo más probable es que el jefe de la expedición nunca se enteró de su existencia.

\section{La descripción y clasificación del trigo recolectado por Boldo en La Habana: el aporte de Mariano Lagasca}

Boldo incorporó a su herbario el trigo de La Habana consciente de que se trataba de un Triticum. La colecta de ese ejemplar tuvo para Bol-

\footnotetext{
${ }^{9}$ Moreno Fraginals, M. (1978), p. 101.
} 
do un mérito indiscutible que fue compartido por José Estévez luego de que éste organizara el herbario de la expedición al ocurrir la muerte de su maestro el 31 de julio de 1799 .

Estévez conservó ese ejemplar de trigo como parte del material botánico que trasladó a España en 1802 para entregarlo al nuevo director del Real Jardín Botánico de Madrid, José Antonio Cavanilles. Sin embargo, en uno de los trabajos más importantes de la expedición, la Cubensis Prima Flora de Boldo y Estévez no aparece ningún Triticum. Así lo hacen constar los índices de nombres vulgares y de especies de la primera edición de esa monumental obra en $1990^{10}$, lo cual confirma que no todas las plantas colectadas por Boldo aparecen incluidas en la Flora.

Debido a esa omisión, el único ejemplar de trigo que había sido recolectado por Boldo en Cuba corrió el riesgo de haber pasado desapercibido entre las numerosas plantas del herbario de la expedición, de no haber efectuado Cavanilles la distribución de esas plantas en diversas secciones del Jardín ${ }^{11}$. Esto propició que su antiguo alumno Mariano Lagasca tuviera acceso a ese ejemplar de trigo de La Habana.

Los estudios de experimentación en fisiología vegetal de Lagasca estuvieron muy marcados por la sólida formación taxonómica y sistemática que recibiera de su maestro Cavanilles. En unión de Simón de Rojas Clemente, Lagasca pretendió estudiar diferentes variedades cultivadas para obtener de ellas dos tipos de ventajas:

- las agronómicas (conocimiento de variedades existentes, posibilidad de seleccionar las más adecuadas a cada condición o las más productivas).

- las sistemáticas (estudio del origen de la variabilidad).

A esa doble ventaja se adscribía el programa agrobotánico que esperaban ofrecer Lagasca y Clemente en relación con la "Ceres Española» y «Ceres Europea».

Además de garantizar la recolección de todas las variedades de cereales cultivadas en España y en Europa con la creación de una extensa red de corresponsales y suministradores, el programa de investigación cerealero contemplaba, en opinión de Joaquín Fernández, lo siguiente:

\footnotetext{
10 Véase: Fernández Casas, J.; Puig-Samper, M. A. y Sánchez García, F. J. (eds.) (1990), «Cubensis Prima Flora...», Fontqueria 29, pp. 195-201.

11 Herrera Oliver, P. P. (1993) p. 156. 


\section{ROLANDO E. MISAS JMÉNEZ}

... clasificación sistemática dando nombres a las distintas variedades o razas encontradas; siembras sistemáticas de los granos (primero en el Real Jardín Botánico de Madrid y más tarde en diversos jardines de Londres y de la isla de Jersey), que se recibían en los envíos y análisis de su capacidad germinativa; tiempo de germinación y detalles de su crecimiento; formación de un herbario completo de dichas variedades y redacción de una obra descriptiva y sistemática donde se incluirían los experimentos realizados y las características particulares de cada variedad ${ }^{12}$.

En el contexto de investigación cerealera que realizaba Lagasca en el Real Jardín Botánico de Madrid encontró el ejemplar de trigo que había depositado Cavanilles como parte de la distribución del herbario de Boldo. A pesar de que prestaba una atención preferencial a las variedades de trigo de España y de Europa, no dejó de despertar su curiosidad científica la presencia del trigo habanero.

Lagasca lo adscribió al género Triticum y lo situó, con decisión y seguridad, junto a Polonicum. Sin embargo, como difería en algo de esta especie por presentar un tallo frutescente o perenne y una espiga semejante a la forma que él denominaba T. aestivum, Lagasca decidió incluir el ejemplar de Boldo en una nueva especie $T$. spinulosum, que describió en los siguientes términos:

Espiguillas 4-5 flores, flores interiores hermafroditas, aristadas: glumas aristadas. Tallo frutescente. Triticum Boldo Herb, Tallo frutescente, envuelto inferiormente por las vainas de las hojas dísticas. Hojas algo rígidas, planas, acuminadas, subpungentes, denticulado-espinosas, las superiores eminentes. Espiga semejante al T. aestivum, casi de dos pulgadas, Hab. en Habana y D. Balth la recolectó para Boldo ${ }^{13}$.

El hecho de que Lagasca definiera ese ejemplar como una nueva especie demuestra que debió sorprenderse ante la colecta realizada por Boldo en Cuba. Debió considerarlo algo excepcional dada la circunstancia de ser un ejemplar único que había crecido en un medio natural supuesta-

\footnotetext{
12 Fernández Pérez, J. (s. a.)»Ciencia y Técnica en la Agricultura Ilustrada. Instrumentos y experiencias Agronómicas», en: Fernández Pérez, J.; González Tascón, I. (eds.), Ciencia, Técnica y Estado en la España Ilustrada. Zaragoza, pp. 65-66.

13 MisAs JimÉnEZ, R. E. (1991), p. 124.
} 
mente desfavorable al cultivo de ese cereal desde que así lo expresara, en 1590, el jesuita Joséph de Acosta ${ }^{14}$. En definitiva, optó por definir el ejemplar cubano como una nueva especie de Triticum, dejándose llevar por las dudas que le embargaban ante una flora desconocida y lejana.

Lagasca no pudo realizar un estudio más completo de la especie Polonicum ni tampoco pudo concluir su soñada Ceres Española ya que su labor en el Real Jardín Botánico de Madrid fue interrumpida durante la guerra contra los invasores franceses y el exilio en Inglaterra. Por consiguiente, su atención hacia el trigo que recolectara Boldo en Cuba se limitó a la descripción y clasificación, lo cual no impidió que quedara esa constancia documental en el más completo olvido hasta 1952.

Al parecer, Lagasca fue el último botánico que vio personalmente ese ejemplar de trigo. En la revisión que realizaron en 1952 los ingenieros agrónomos Ricardo Téllez y Manuel Alonso de los herbarios y descripciones de trigo que fueron utilizados por Lagasca para su malogrado Ceres, dedicaron una particular atención a la búsqueda de ese trigo y de otros datos referentes al mismo, lo cual dio el siguiente resultado:

Ni en el herbario de trigos, ni en el general del Jardín Botánico, ni en los particulares que se conservan en dicho centro madrileño, hemos encontrado tan curioso ejemplar de trigo. Tampoco volvemos a encontrar citada la curiosa especie $\mathrm{T}$. spinulosum en ninguna obra ni manuscrito de Clemente o de Lagasca (...) El Index londinensis, tomándola de Lagasca, cita esta especie, pero no da ninguna otra sinonimia, indicación o referencia. No podemos dar nuestra opinión sobre $\mathrm{T}$. spinulosum, y, no figurando en el herbario, lo omitimos en esta revisión ${ }^{15}$.

\section{La vigencia en Cuba del aporte de Boldo y Lagasca}

La falta de ese ejemplar de trigo fue hasta cierto punto compensada con la descripción y clasificación que le hiciera Lagasca. Sin embargo, ese aporte científico de Lagasca no parece haber sido conocido en Cuba

\footnotetext{
14 Acosta, Joséph de (1894) Historia Natural y Moral de las Indias exrita por el P. Joséph de Acosta de la compañia de Jesús. Publicada en Sevilla en 1590 y ahora fielmente reimpresa en la primera edición, Madrid, pp. 360-361.

15 Misas Jiménez, R. E. (1991), pp. 124-125. 


\section{ROLANDO E. MISAS JIMÉNEZ}

ni aún después de haberlo publicado en Madrid, en 1952, los ingenieros Téllez y Alonso en su importante libro.

A pesar de lo útil que hubiera sido disponer de esa información para que el trigo se cultivara de manera definitiva en la isla, en realidad la carencia de un serio esfuerzo de indagación histórica hacia los problemas de la tecnología agrícola conspiró contra esa necesidad. Aún no se había profesionalizado ese tipo de investigación histórica.

Estudios actuales han permitido que la descripción y clasificación que hiciera Lagasca del trigo encontrado por Boldo en La Habana sirviera para confirmar la sospecha que tenía en 1848 el humanista cubano Antonio Bachiller y Morales de que la variedad de trigo de La Habana era la misma que la variedad «barbada» de Villa Clara. Independientemente de las diferencias que habían entre el conocimiento científico de Lagasca sobre el trigo de La Habana y las sencillas observaciones morfológicas realizadas por Bachiller con el trigo de Villa Clara, ambas personalidades coincidieron en otorgarle a sus respectivos trigos rasgos semejantes a la especie Polonicum ${ }^{16}$.

En definitiva, las contribuciones de Boldo, Lagasca y Bachiller han demostrado la vigencia que tiene en Cuba esos lejanos esfuerzos al vincularse a las actuales instituciones agronómicas dedicadas a los experimentos en trigo.

16 Misas JimÉnez, R. E. (1991), p. 125. 